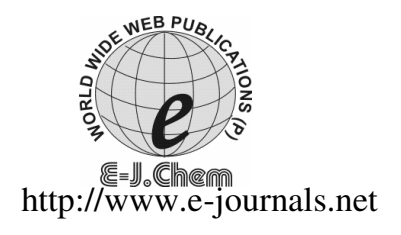

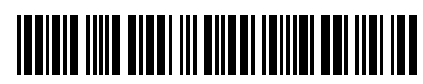

ISSN: 0973-4945; CODEN ECJHAO

E-Journal of Chemistry 2012, 9(1), 175-180

\title{
Synthesis and Biological Activity of Some 3,4-Dihydro-4-(4-substituted aryl)-6-(naptho [2,1-b]furan-2-yl pyrimidine-2(1H)-one Derivatives
}

\author{
SANJEEVAN S. GAIKWAD, VENKAT S. SURYAWANSHI ${ }^{\S *}$, \\ KISHAN S. LOHAR, DHANAJI V. JADHAV ${ }^{\#}$ and NARAYAN D. SHINDE ${ }^{\S}$ \\ Dept. of Chemistry, Shrikrishna College, Gunjoti \\ Tal-Omerga, Dist-Osmanabad, Maharashtra-413613, India \\ ${ }^{\S}$ Dept. of Chemistry, PG studies and Research Center \\ Shri Chattrapati Shivaji College, Omerga, Maharashtra-413 606, India \\ ${ }^{\#}$ Dept. of Chemistry, Y. C. Arts \& Science College, Mangrulpir \\ Dist-Washim, Maharashtra-444 403, India \\ venkatesh1@sify.com
}

Received 25 June 2011; Accepted 13 August 2011

\begin{abstract}
A series of new oxopyrimidine were prepared by cyclocondensation route with various substituted chalcones in presence of alcoholic solution of potassium hydroxide at reflux temperature. The synthesized oxopyrimidine derivatives were characterized by means of their IR, ${ }^{1} \mathrm{H}$ NMR, mass spectral data and elemental analysis. The synthesized oxopyrimidines derivatives were evaluated for antibacterial and antifungal activities, some of them were found to possess significant activity.
\end{abstract}

Keywords: Naphthofuran, Chalcone, Oxopyrimidines, Synthesis, Biological activities

\section{Introduction}

The search for new potent anti-microbial agents with reduced toxicity and lower side effects is of continuous process. One of the most frequently encountered groups of organic compound in medicinal chemistry is oxopyrimidine and their derivatives. Pyrimidines ${ }^{1}$ have occupied unique place in the field of medicinal chemistry. Some antibacterial ${ }^{2-7}$ and antimalerial ${ }^{8-10}$ drug is constituted by pyrimidine derivatives. Certain pyrimidine derivatives are known to display as analgesic ${ }^{11}$, anthelmintic ${ }^{12-13}$, antitumor ${ }^{14}$, antifungal ${ }^{15}$, antiviral ${ }^{16-18}$, anticancer $^{19}$, insecticidal ${ }^{20}$ and diuretic $\&$ anti-inflammatory ${ }^{21-22}$ activities. Keeping in view of the biological importance ${ }^{23}$ and chemotherapeutic properties of oxopyrimidine and naphthofuran derivatives, attempts has been made to synthesize some new oxopyrimidine derivatives linked with naphthofuran and aryl group with the hope that they might display antimicrobial activity. 


\section{Experimental}

Melting points were determined on an open capillary melting point apparatus and are uncorrected. IR spectra were recorded on Brucker FT-IR (Alpha-P) spectrometer, ${ }^{1} \mathrm{H}$ NMR spectra were recorded in $\mathrm{CDCl}_{3}$ on Bruker "AVANCE 400" $\mathrm{MHz}$ spectrometer using TMS as an internal standard and Mass spectrum on Shimadzu GCMS QP 5050A,modeDI,operating at $70 \mathrm{eV}$. Progress of reaction was monitored by TLC. Naphthaldehyde, chloroacetone, Urea, aromatic aldehydes were purchased from Merk, India. All compounds have been recrystallized in ethanol.

\section{Synthesis of 2-acetylnaphtho [2, 1-b] furan (1)}

In a $250 \mathrm{~mL}$ four necked round bottom flask fitted with overhead mechanical stirrer, a dropping funnel, a thermometer and condenser with child water circulation, 2-hydroxy-1naphthaldehyde $(0.1 \mathrm{~mole})$, chloroacetone $(0.11 \mathrm{~mole})$ and anhydrous potassium carbonate $(15 \mathrm{gm})$ were refluxed in dry acetone $(75 \mathrm{~mL})$ for $12 \mathrm{~h}$. Potassium salts were filtered off, the filtrate on removal of solvent and on trituration with ethanol gave the pale yellow crystals of 2-acetylnaphtho [2, 1-b] furan (1). The sample was purified by recrystallized from absolute ethanol. $\mathrm{MP}=96^{\circ} \mathrm{C}$, Yield $=60 \%$

Typical synthesis of 3-(4-hydroxyphenyl)-1 (naphtho [2,1-b]furan-2 yl) prop-2-en1-one (2c)

A mixture of 2-acetylnaphtho [2,1-b] furan (1) (0.02 mole) and p-hydroxy benzaldehyde $(0.022$ mole) was stirred in ethanol $(50 \mathrm{~mL})$ and then in aqueous solution of potassium hydroxide $(50 \%)(10 \mathrm{~mL})$ was added to it portion wise, keeping the temperature below $10{ }^{\circ} \mathrm{C}$ throughout the addition. The mixture was kept for $36 \mathrm{~h}$ and it was acidified with conc. $\mathrm{HCl}$. The reaction mixture was poured into crushed ice and the solid obtained was filtered under vaccum. It was washed firstly with sodium carbonate solution and then with water, dried and the product was recrystalized from ethanol. Same procedure is extended for other compounds of this series. (2a-e) were synthesized by using appropriate aldehyde. The physical data of chalcones were shown in Table 1.

Table 1. Physical data of the synthesized compounds

\begin{tabular}{|c|c|c|c|c|c|c|c|c|}
\hline \multirow{4}{*}{$\begin{array}{c}\text { Compound } \\
\text { No. }\end{array}$} & \multirow{4}{*}{$\begin{array}{l}\text { Molecular } \\
\text { Formula }\end{array}$} & \multirow{4}{*}{$\begin{array}{c}\text { Molecular } \\
\text { Weight }\end{array}$} & \multirow{4}{*}{$\begin{array}{c}\text { Yield } \\
\%\end{array}$} & \multirow{4}{*}{${ }^{\mathrm{M}} \mathrm{C}$ C.P. } & \multicolumn{4}{|c|}{ Elements \% } \\
\hline & & & & & $\mathrm{C}$ & $\mathrm{H}$ & $\mathrm{N}$ & $\mathrm{Cl}$ \\
\hline & & & & & Calcd. & Calcd & Calcd & Calcd \\
\hline & & & & & Found & Found & Found & Found \\
\hline \multirow{2}{*}{$2 \mathbf{a}$} & \multirow{2}{*}{$\mathrm{C}_{21} \mathrm{H}_{14} \mathrm{O}_{2}$} & \multirow{2}{*}{298.1} & \multirow{2}{*}{60} & \multirow{2}{*}{132} & 84.53 & 4.69 & - & - \\
\hline & & & & & 84.55 & 4.7 & - & - \\
\hline \multirow{2}{*}{$2 b$} & \multirow{2}{*}{$\mathrm{C}_{22} \mathrm{H}_{16} \mathrm{O}_{2}$} & \multirow{2}{*}{312.1} & \multirow{2}{*}{66} & \multirow{2}{*}{151} & 84.58 & 5.12 & - & - \\
\hline & & & & & 84.57 & 5.10 & - & - \\
\hline \multirow{2}{*}{$2 c$} & \multirow{2}{*}{$\mathrm{C}_{21} \mathrm{H}_{14} \mathrm{O}_{3}$} & \multirow{2}{*}{314.0} & \multirow{2}{*}{65} & \multirow{2}{*}{172} & 80.25 & 4.45 & - & - \\
\hline & & & & & 80.24 & 4.44 & - & - \\
\hline \multirow{2}{*}{ 2d } & \multirow[t]{2}{*}{$\mathrm{C}_{23} \mathrm{H}_{16} \mathrm{O}_{3}$} & \multirow{2}{*}{340.0} & \multirow{2}{*}{72} & \multirow{2}{*}{135} & 81.17 & 4.70 & - & - \\
\hline & & & & & 81.11 & 4.69 & - & - \\
\hline \multirow{2}{*}{$2 e$} & \multirow[t]{2}{*}{$\mathrm{C}_{21} \mathrm{H}_{13} \mathrm{ClO}_{2}$} & \multirow{2}{*}{332.7} & \multirow{2}{*}{55} & \multirow{2}{*}{143} & 75.74 & 3.90 & - & 10.67 \\
\hline & & & & & 75.73 & 4.00 & - & 10.61 \\
\hline
\end{tabular}


Synthesis of 3, 4-dihydro-6-(naphtho [2,1-b]furan-2-yl)4-phenyl pyrimidine-2(1H)one (3a-e)

A mixture of 1-(naphtho [2, 1-b] furan-2-yl)-3-phenyl prop-2-en-1-one (2a) (0.01 mole) and urea $(0.02 \mathrm{~mole})$ dissolved in ethanol $(50 \mathrm{~mL})$ under stirring. To this an alcoholic potassium hydroxide $50 \%(10 \mathrm{~mL})$ was added and refluxed for $10 \mathrm{~h}$. After completion of reaction, it was cooled and poured in ice cold water and neutralized by acetic acid. The solid product was filtered washed with water and recrystallized from absolute ethanol. Compounds (3b-e) were prepared similarly from (2b-e). The physical data of oxopyrimidine derivatives are given in Table 2.

Table 2. Physical data of the synthesized compounds

\begin{tabular}{|c|c|c|c|c|c|c|c|c|}
\hline \multirow{4}{*}{ Compound } & \multirow{4}{*}{$\begin{array}{l}\text { Molecular } \\
\text { Formula }\end{array}$} & \multirow{4}{*}{$\begin{array}{c}\text { Molecular } \\
\text { Weight }\end{array}$} & \multirow{4}{*}{$\begin{array}{c}\text { Yield } \\
\%\end{array}$} & \multirow{4}{*}{${ }^{\mathrm{M}} \mathrm{C} . \mathrm{P}$} & \multicolumn{4}{|c|}{ Elements \% } \\
\hline & & & & & $\mathrm{C}$ & $\mathrm{H}$ & $\mathrm{N}$ & $\mathrm{Cl}$ \\
\hline & & & & & Calcd. & Calcd & Calcd & Calcd \\
\hline & & & & & Found & Found & Found & Found \\
\hline \multirow{2}{*}{$3 \mathbf{a}$} & \multirow{2}{*}{$\mathrm{C}_{22} \mathrm{H}_{16} \mathrm{~N}_{2} \mathrm{O}_{2}$} & \multirow{2}{*}{340.3} & \multirow{2}{*}{52} & \multirow{2}{*}{235} & 77.57 & 4.7 & 8.22 & - \\
\hline & & & & & 77.56 & 4.7 & 8.21 & - \\
\hline \multirow{2}{*}{$\mathbf{3 b}$} & \multirow{2}{*}{$\mathrm{C}_{23} \mathrm{H}_{18} \mathrm{~N}_{2} \mathrm{O}_{2}$} & \multirow{2}{*}{354.4} & \multirow{2}{*}{55} & \multirow{2}{*}{247} & 77.87 & 5.07 & 7.90 & - \\
\hline & & & & & 77.81 & 5.00 & 7.89 & - \\
\hline \multirow{2}{*}{$3 c$} & \multirow[t]{2}{*}{$\mathrm{C}_{22} \mathrm{H}_{16} \mathrm{~N}_{2} \mathrm{O}_{3}$} & \multirow{2}{*}{356.12} & \multirow{2}{*}{56} & \multirow{2}{*}{297} & 74.13 & 4.49 & 7.86 & - \\
\hline & & & & & 74.19 & 4.48 & 7.86 & - \\
\hline \multirow{2}{*}{ 3d } & \multirow[t]{2}{*}{$\mathrm{C}_{23} \mathrm{H}_{18} \mathrm{~N}_{2} \mathrm{O}_{3}$} & \multirow{2}{*}{370.1} & \multirow{2}{*}{52} & \multirow{2}{*}{260} & 74.57 & 4.86 & 7.56 & - \\
\hline & & & & & 74.49 & 4.85 & 7.55 & - \\
\hline \multirow{2}{*}{$3 e$} & $\mathrm{C}_{22} \mathrm{H}_{15}$ & \multirow{2}{*}{374.08} & \multirow{2}{*}{50} & \multirow{2}{*}{258} & 70.43 & 4.0 & 7.47 & 9.47 \\
\hline & $\mathrm{ClN}_{2} \mathrm{O}_{2}$ & & & & 70.10 & 4.04 & 7.46 & 9.49 \\
\hline
\end{tabular}

\section{Purification}

Crude product was dissolved in $10 \mathrm{~mL}$ absolute ethanol and heated up to $70{ }^{\circ} \mathrm{C}$ to get clear solution and cooled slowly up to $10{ }^{\circ} \mathrm{C}$, filtered, sucked and dried in vacuum to offered pure compound. Under similar condition, a compound of this series has been carried out. Results are summarized in Table $1 \& 2$. Good to excellent yields and perfect selectivity was obtained in all cases.

\section{Spectral Discussion}

\section{Compound $2 c$}

IR ( $\left.\mathrm{KBr}, \lambda_{\max }\right): 3310 \mathrm{~cm}^{-1}$ (Ar-O- H str.), $3058 \mathrm{~cm}^{-1}$ (-CH str. of Ar), $1644 \mathrm{~cm}^{-1}$ (C=O str. in ketone), $1586 \mathrm{~cm}^{-1}(\mathrm{C}=\mathrm{C}), 1515 \mathrm{~cm}^{-1}(\mathrm{C}=\mathrm{C}$ str. in $\mathrm{Ar}), 1443 \& 1359 \mathrm{~cm}^{-1}\left(\mathrm{CH}_{3}\right.$ def. $), 1153$ \& $1167 \mathrm{~cm}^{-1}$ (C-O-C str.), $830 \mathrm{~cm}^{-1}$ (-CH str.), $747 \mathrm{~cm}^{-1}$ (Ar-H opb.) ${ }^{1} \mathrm{H}$ NMR (CDCl 3 in Sppm) $6.35(\mathrm{~d}, 1 \mathrm{H},-\mathrm{CO}-\mathrm{CH}=), 6.95(\mathrm{~d}, 1 \mathrm{H}, \mathrm{C}=\mathrm{CH}), 7.21-8.24$ (complex m, Ar. Proton $11 \mathrm{H}), 10.32(\mathrm{~s}, 1 \mathrm{H}$, phenolic $-\mathrm{OH})$ proton. Mass $(\mathrm{m} / \mathrm{z}): 314[\mathrm{M}]^{+}, 221,195,147,119,118$, $91,69,65,43$.

\section{Compound 3a}

IR $\left(\mathrm{KBr}, \lambda_{\max }\right)$ : $3300-3400 \mathrm{~cm}^{-1}(\mathrm{C}-\mathrm{H}$ str. in $\mathrm{Ar}), 1659 \mathrm{~cm}^{-1}(\mathrm{C}=\mathrm{O}$ str. in N-CO-N$)$, $1541 \mathrm{~cm}^{-1}$ (-C-O-C- in Ar), $1262 \mathrm{~cm}^{-1}$ (C-O-C str.) ${ }^{1} \mathrm{H} \mathrm{NMR}\left(\mathrm{CDCl}_{3}\right.$ in $\left.\delta \mathrm{ppm}\right) 5.46$ (d, 1H, H-4), 5.68 (s, 1H, N-H-3), 5.84(d, 1H, H-5), 6.01(s, 1H, N-H-1), 6.71-7.80 (m, 12H, Ar-H) Mass $(\mathrm{m} / \mathrm{z}): 340[\mathrm{M}]^{+}, 297,296,220,198,197,194,105,104,103,102$, 101, 91, 77, 70 . 


\section{Reaction Scheme}<smiles>CC(=O)c1cc2c(ccc3ccccc32)o1</smiles><smiles>O=Cc1ccc([R]O[Na])cc1</smiles><smiles>[R]c1ccc(/C=C/C(=O)c2cc3c(ccc4ccccc43)o2)cc1</smiles>
Reflux $\mid \begin{aligned} & 2 \mathrm{a}-2 \mathrm{e} \\ & \mathrm{NH}_{2} \mathrm{CONH} \\ & \mathrm{KOH} / \mathrm{C}_{2} \mathrm{H}_{5} \mathrm{OH}\end{aligned}$<smiles></smiles>

$3 a-3 e$

\section{Scheme 1}

$R=$ Substituted group $R=a=4-H, R=b=4-C_{3}, R=c=4-O H, R=d=4-O_{C H}, R=e=4-C l$.

\section{Antimicrobial activity}

The antimicrobial activity was determined by cup-plate method ${ }^{24-25}$. In vitro antimicrobial activity was carried out against $24 \mathrm{~h}$. culture of four bacteria and four fungi. The bacteria used were Escherichia coli, Salmonua typhi, Staphylococcus aureus and Bacillus substilis. While the fungi used were Aspergillus niger, Penicillium chrysogenum, Fusirium moneliforme and Candida albicans. The compounds were tested at a $(20 \mathrm{mg})$ dissolved in dimethyl sulphoxide $(5 \mathrm{~mL})$ against all organisms. Penicillin and griseofulvin were used as standards for comparison of antibacterial and antifungal activities respectively. The zone of inhibition was compared with the standard drug after $24 \mathrm{~h}$. incubation at $37{ }^{\circ} \mathrm{C}$ for antibacterial activity $\& 48 \mathrm{~h}$. at $28-30{ }^{\circ} \mathrm{C}$ for antifungal activity. The results are reported in Table 3. 
Table 3. Antimicrobial activity of synthesized compounds

\begin{tabular}{|c|c|c|c|c|c|c|c|c|}
\hline \multirow{2}{*}{ Compound } & \multicolumn{4}{|c|}{$\begin{array}{l}\text { Antibacterial activity } \\
\text { zone of inhibition }\end{array}$} & \multicolumn{4}{|c|}{ Antifungal activity } \\
\hline & E.coli & S.typi & S.aureus & $\begin{array}{l}\text { B.sub } \\
\text { stilis }\end{array}$ & A.niger & $\begin{array}{c}P . \\
\text { Chrysogen. }\end{array}$ & $\begin{array}{c}F . \\
\text { Moneli }\end{array}$ & $\begin{array}{c}C . \\
\text { Albicans }\end{array}$ \\
\hline $\mathbf{3 a}$ & -ve & -ve & 13 & -ve & -ve & $-\mathrm{ve}$ & +ve & -ve \\
\hline $3 b$ & -ve & -ve & 14 & 11 & $+v e$ & -ve & -ve & $+v e$ \\
\hline $3 c$ & -ve & -ve & 13 & 11 & -ve & $+v e$ & $+v e$ & -ve \\
\hline 3d & -ve & -ve & 12 & -ve & $+v e$ & $+v e$ & -ve & -ve \\
\hline $\mathbf{3 e}$ & -ve & -ve & 15 & 15 & -ve & $+v e$ & -ve & $+v e$ \\
\hline penicillin & 18 & 20 & 32 & 28 & - & - & - & - \\
\hline Griseofulvin & - & - & - & - & +ve & +ve & $+v e$ & $+v e$ \\
\hline
\end{tabular}

Penicillin \& Griseofulvin were used as standards for antibacterial \& antifungal activities respectively. Control (DMSO) - ve no activity

\section{Results and Discussion}

Compounds (3a-e) showed significant antibacterial activity at $0.02 \mathrm{~mL}(20 \mathrm{mg})$ concentration level when compared with standard drug penicillin. However the oxopyrimidine $\mathbf{3 b}, \mathbf{3 c} \& \mathbf{3} \mathbf{e}$ were found to be more potent on all the bacteria strains. Compounds (3a-e) also showed significant antifungal activity at $0.02 \mathrm{~mL}(20 \mathrm{mg})$ concentration levels when compared with standard drug griseofulvin compound $\mathbf{3 a}, \mathbf{3 b}, \mathbf{3 c}$, 3d \& 3e showed maximum antifungal activity.

\section{Acknowledgment}

The authors are thankful to Dr.C.H.Gill, Dr. M.S.Shingare, Dr. R.A.Mane Dept.of chemistry, Dr.D.R.Mane, Dean, Faculty of Science, Dr. B.A.M. University, Aurangabad for providing necessary facilities and helpful suggestions.

\section{References}

1. Maria T C, Cenzo Congiu, Antonio Maccioni and Valentina Onnis, J Het Chem., 2009, 31(2), 329.

2. Mahadevan K M and Vaidya V P, Indian J Pharm Sci., 2003, 65, 128.

3. Burger A, Medicinal Chemistry, Wiley Inter Science., New York, 1961.

4. Vagdevi H.M, Latha K P, Vaidya V P and Vijay Kumar M.L, Indian J Pharm Sci.., 2001, 63(4), 286.

5. Foye W O, Principles of Medicinal Chemistry, Lea and Febiger, Philadelphia, 1981.

6. Pershin G N, Sherbakova L I and Zykova T N., Sakolova Farmakol aksikol, 1972, 35, 466-471, Chem Abstr., 1972, 77, 1555802.

7. Kodihalli C R, Hosadu M V and Vijayvithal P V, ARKIVOC, 2008, XI, 1-10.

8. Steck E A, Chemotherapy of protozoan Diseases; Walter Reed, Army Institute of Res., Washington D.C., 1971.

9. Thompson P E and Werbal L M, Antibacterial Agents Chemistry Pharmacology, Academic press, New York, 1972.

10 Falco E A and Brit J Pharmacol., 1961, 6, 185.

11 Pemmisin M, Lue-Due C, Hoguet F and Gaultier C, Narcisse J, Eur J Chem., 1988, 23, 534 .

12 Ram V J, Pak J Chem., 1989, 331, 893. 
13 Ram V J, Haque N and Guru P Y, Eur J Med Chem., 1992, 27, 851-855.

14 Suguira K, Schimid M M and Brown FG, Cancer Chemother Rep., 1973, 2, 231-233.

15 Basavraj P, Vaidya V P and Vijay Kumar M L, Indian J Heterocycl Chem., 2002, 12, 89-94.

16 Balzarini J and McGuigan C, J Antimicrob Chemothr., 2002, 50(1), 5-9.

17 Mercer F L, Lindhorst L E and Commoner B, Science, 1953, 117, 558.

18 Nasr M.N and Gineinah M M, Arch Pharm., 2002, 335, 289-295.

19 EI-Gaby M S, Abdel-Hamide S G and Ghorab M M and El-Sayed S M, Acta Pharm., 1999, 49, 149.

20 Cheng C C, Progr Med Chem., 1969, 6, 67-134.

21 Kumarswamy M N, Prathima Matthias D A, Chandrasekhar C and Vaidya V P, Indian J Pharm Sci., 2006, 68(6), 731-736.

22 Nega S, Aionso J, Diazj A and Junquere F, J Het Chem., 1990, 27, 269.

23 Sondhi S M, Johar M, Rajvanshi S, Dastidar S G, Australian J Chem., 2001, 54, 69-74.

24 Banty A L, Antimicrobial susceptibility tests: Principle and practice; Edited by Illus, Lea and Febiger, Philadelphia, PA: USA, 1976,180.

25 Saundane A R, Rudresh K, Satyanarayan N D and Hiremath S.P, Indian J Pharm Sci., 1998, 60(6), 379-383. 


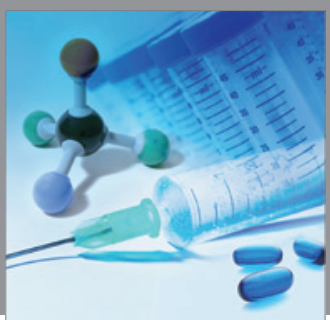

International Journal of

Medicinal Chemistry

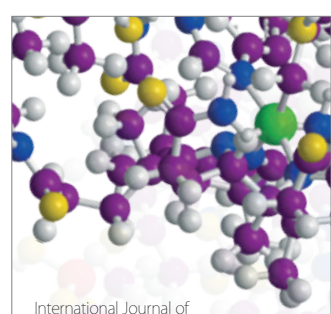

Carbohydrate Chemistry

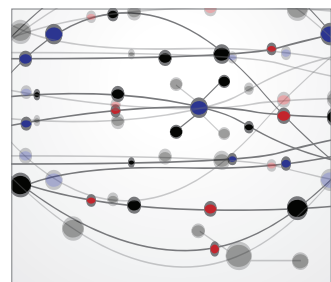

The Scientific World Journal
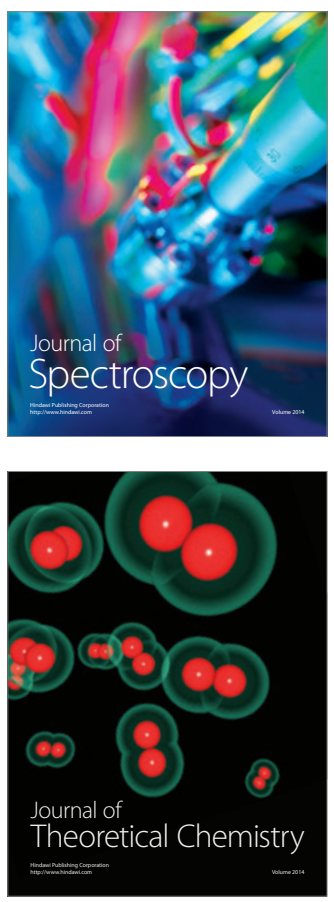
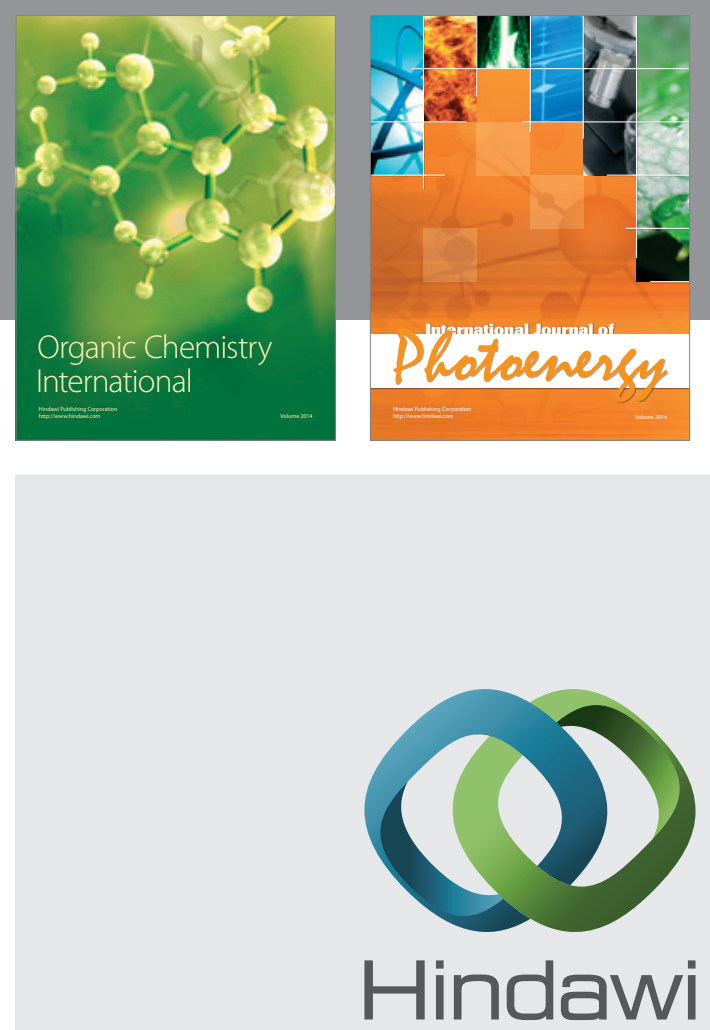

Submit your manuscripts at

http://www.hindawi.com
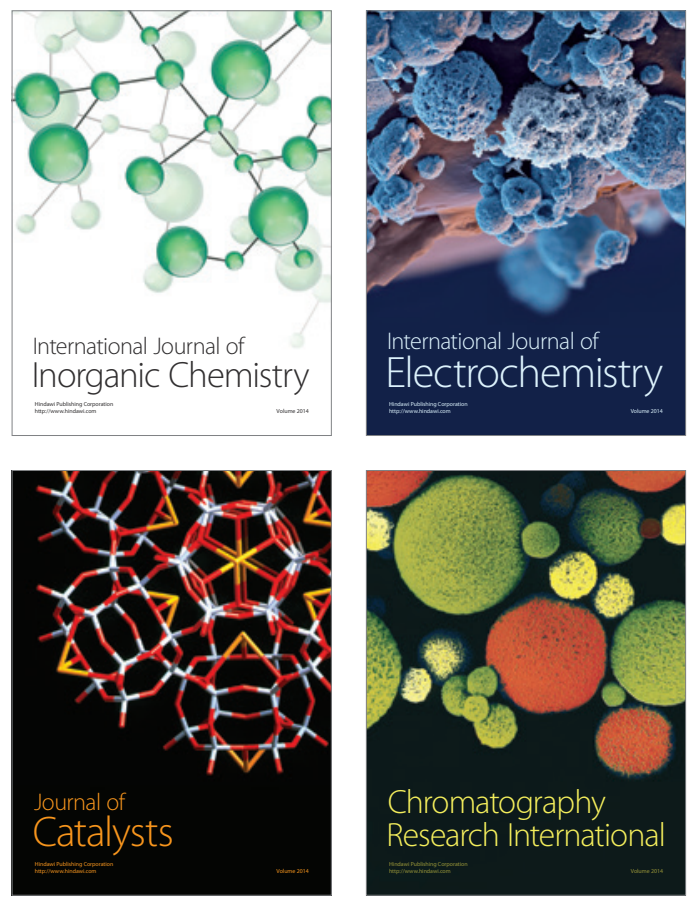
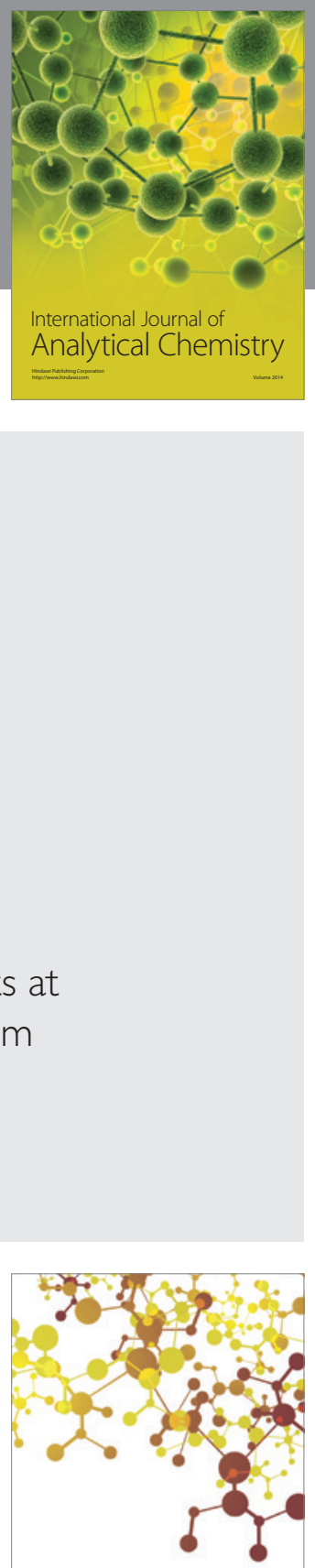

Journal of

Applied Chemistry
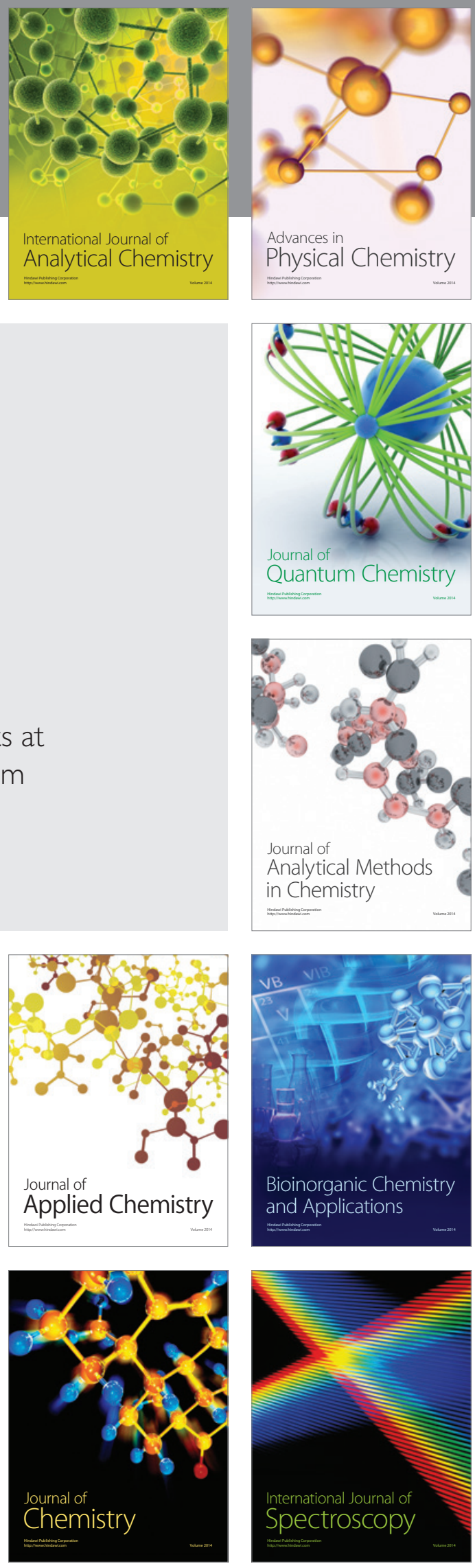